\title{
Assessing Plant Diversity in a Dry Tropical Forest: Comparing the Utility of Landsat and Ikonos Satellite Images
}

\section{Harini Nagendra ${ }^{1,2}, *$, Duccio Rocchini ${ }^{3}$, Rucha Ghate ${ }^{4}$, Bhawna Sharma ${ }^{1}$ and Sajid Pareeth ${ }^{1}$}

1 Ashoka Trust for Research in Ecology and the Environment (ATREE), Royal Enclave, Srirampura, Jakkur Post, Bangalore 560064, Karnataka, India; E-Mails: bhawna01@ gmail.com (B.S.); spareeth@gmail.com (S.P.)

2 Center for the Study of Institutions, Population, and Environmental Change (CIPEC), Indiana University, 408 N. Indiana Ave., Bloomington, IN 47408, USA

3 IASMA Research and Innovation Centre, Fondazione Edmund Mach, Environment and Natural Resources Area, Via E. Mach 1, 38010 S. Michele all'Adige, TN, Italy;

E-Mail: duccio.rocchini@iasma.it (D.R.) or ducciorocchini@gmail.com (D.R.)

4 SHODH: The Institute for Research and Development, 50, Puranik Layout, Bharat Nagar, Nagpur 440033, Maharashtra, India; E-Mail: ruchaghate@gmail.com

* Author to whom correspondence should be addressed; E-Mail: nagendra @indiana.edu; Tel.: +91-802-583-8081; Fax: +91-802-353-0070.

Received: 15 December 2009; in revised form: 8 January 2010 / Accepted: 28 January 2010 / Published: 2 February 2010

\begin{abstract}
While high expectations have been raised about the utility of high resolution satellite imagery for biodiversity assessment, there has been almost no empirical assessment of its use, particularly in the biodiverse tropics which represent a very challenging environment for such assessment challenge. This research evaluates the use of high spatial resolution (IKONOS) and medium spatial resolution (Landsat ETM+) satellite imagery for assessing vegetation diversity in a dry tropical forest in central India. Contrary to expectations, across multiple measures of plant distribution and diversity, the resolution of IKONOS data is too fine for the purpose of plant diversity assessment and Landsat imagery performs better.
\end{abstract}

Keywords: biodiversity assessment; dry tropical forest; India; spatial resolution 


\section{Introduction}

Much expectation has been raised about the utility of high resolution satellite imagery for ecological research [1-3], yet so far the hype has largely outpaced actual field assessments. This is particularly the case in the tropics. Most remote sensing studies of biodiversity concentrate on trees and larger plants which can be more readily discriminated using remote sensors. In areas which have a few dominant species, such as temperate forests or mangroves, hyperspatial data have been used to delineate tree canopies and directly identify individual trees at the species level [4-6]. This task, challenging enough when there are a few species, becomes close to impossible when the number of species approaches the level of tens or hundreds. Such is the case in the tropics, where the higher numbers of species, greater numbers of individuals and increased species and habitat heterogeneity make the task of biodiversity assessment much more challenging, particularly so when done from the sky [7]. Examining the potential of such datasets for vegetation diversity assessment in the tropics therefore remains a significant challenge and one that has, unfortunately, been insufficiently explored so far.

This is especially ironical considering that tropical habitats are where much of the world's species diversity is concentrated [8-10]. With accelerated increase in tropical forest clearing and declines in diversity taking place across the world, there is an urgent need to identify the locations of biodiversity hotspots, map the distribution of vegetation diversity across different habitats and landscapes, and monitor rates of change over time. Does high resolution satellite data hold greater potential than medium resolution data for this purpose?

One of the major perceived limitations of current, widely used medium resolution satellite remote sensing platforms such as Landsat and SPOT has been that of insufficient spatial resolution. These data are collected at pixel resolutions of a few tens of meters in size, where a single pixel generally covers a number of different individual plants, often belonging to different species. Each pixel corresponds to a mixed field signature averaged across multiple objects, leading to difficulties in species identification. Hence, past efforts to use these satellites for ecological studies such as plant diversity estimation have achieved only moderate success [11]. The major use these datasets have been put to in these habitats is for habitat mapping or land cover/land use research $[6,3,12]$.

High resolution satellite imagery, with pixel sizes of the size of $2-5 \mathrm{~m}$, corresponding well to the size of individual tree crowns, has been declared as having much greater potential for mapping vegetation diversity and distributions $[2,4,13,14]$. In the past decade, the launch of very high spatial resolution satellite sensors like IKONOS, QuickBird, OrbView-3 and the Panchromatic band of IRS LISS-3 have provided researchers with the opportunity to study ecological systems at far greater detail than previously possible. These data have been used in multiple studies for plant diversity assessment in habitats with a smaller number of tree species, such as mangroves, temperate forests and boreal forests (e.g., [15-18]).

Yet the fine spatial resolution provided by these sensors can lead to problems. When pixel dimensions shrink to a point where individual pixels are smaller than the size of individual tree crowns, then pixel-pixel variability increases dramatically. For instance, some pixels may cover a leaf in sunshine while others cover a leaf of the same tree in shade, in gaps between leaves, or even on tree 
bark-making it hard to handle relatively simple tasks like delineating tree canopies, let alone assigning signatures to different species [11].

Further, in comparison to hyperspatial data, medium resolution sensors such as Landsat have a greater number of bands and are able to record additional information in the middle infrared range, which relates to a range of critical plant properties including leaf pigment, water content, and chemical composition, and can be very useful for discriminating tree species [19-22]. Landsat also provides data over a longer period of time than most other remote sensing platforms, which makes it of great use for monitoring programs [14,23].

Thus, there appear to be tradeoffs between the utility of high spatial, low spectral resolution and of medium spatial, medium spectral resolution satellite sensors for ecological studies. Yet an exact understanding of these tradeoffs remains elusive, given the lack of field research [9]. This paper evaluates high spatial resolution ( $1 \mathrm{~m}$ pixel) IKONOS satellite imagery as well as medium spatial resolution (30 m pixel) Landsat ETM+ satellite imagery for plant diversity assessment in the dry tropical forests of central India. Given the rapidity of forest clearing in the dry tropics, coupled with the high biodiversity in these areas, there is a pressing need for identifying best practice approaches to quickly assess plant diversity distributions and monitor changes in these habitats: a need that has been insufficiently met this far $[9,24]$. The research described here represents an important step towards this goal.

\section{Study Area}

The Tadoba Andhari Tiger Reserve (TATR) is a national park and wildlife sanctuary located in central India, in the eastern part of Maharashtra state (Figure 1). The protected area extends over $625 \mathrm{~km}^{2}$, covering a landscape that is largely a matrix of dry tropical forests, interspersed with some grasslands, water bodies and a few small patches of riparian forest alongside streams. The park is drained by two main rivers. The southern section of the part is flat, giving way to gradually undulating topography as one moves northwards. There is a well developed road network in the northeastern part of the reserve, which provides access to the forest for grazing and biomass extraction. To the north, south and east, the TATR has some protection from surrounding State controlled Reserve Forest and Protected Forest areas. During the time of field work, six villages were located within the boundaries of the park (one village has since been relocated outside the park), and there were 53 villages located on the periphery. In addition to the six interior villages, several other villages and communities access resources from the park. These villages fulfil a large part of their fuel, fodder, timber and non-timber forest requirements from the park [25]. The TATR also experiences substantial seasonal use from migrant herders, and is frequented by timber, bamboo and wildlife poachers. Thus, despite being located within a protected area, this dry tropical forest habitat is also subject to human disturbance due to grazing, fire and biomass extraction. While the road network in the northern part of the reserve is quite well developed, providing greater access to the forest and leading to greater disturbance, the TATR is surrounded on the southern and eastern sides by better protected State controlled Reserve Forest and Protected Forest areas. This study area contains a large contiguous region of dry tropical forest habitat, in various stages of protection ranging from the better protected forest core to more degraded areas at the park boundary adjacent to settlements [25]. This region thus provides us with an opportunity to study variation in vegetation diversity and distribution within a relatively 
heterogeneous, large contiguous patch of dry tropical forest: a habitat type which remains little studied despite its importance and susceptibility to change [9,24].

Figure 1. Study area map showing the location of the 211 field plots overlaid on a Normalized Difference Vegetation Index derived from a Landsat ETM+ image of 29th October 2001.

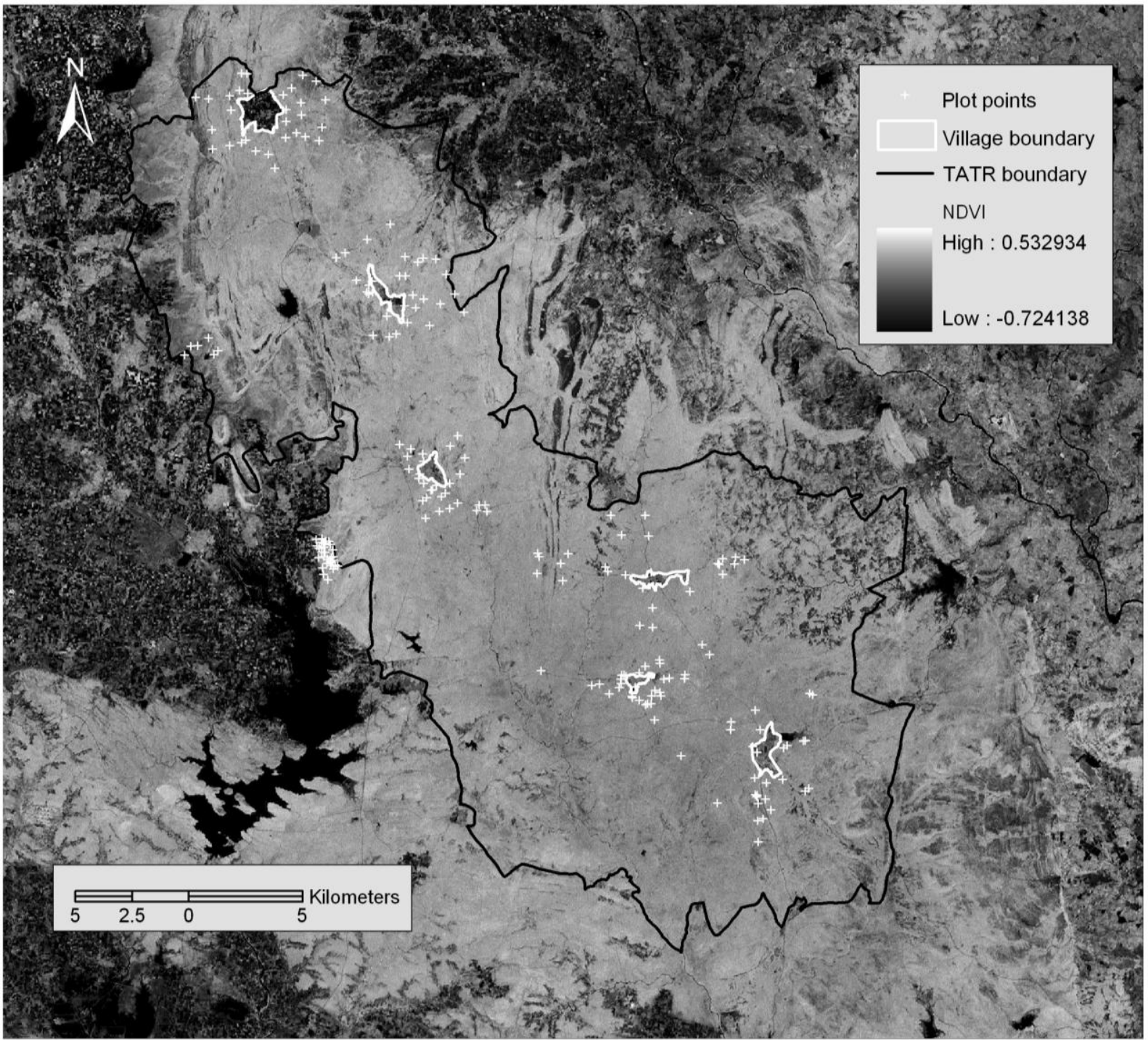

\section{Materials and Methods}

\subsection{Data Gathering}

\subsubsection{Field data collection}

Between 2003 and 2005, a total of 240 circular plots were distributed across the TATR to sample plant biodiversity distributions, taking care to ensure that we sampled plant biodiversity at a range of distances from the settlements within and outside the protected area. Such a sampling strategy thus enables us to sample the variation in biodiversity within the park, from relatively undisturbed core areas to degraded areas adjacent to the park outer boundary. Since some of the plots were located 
outside the landscape covered by the IKONOS images, these were removed from analysis, leaving a total of 211 field plots that were used for this study. Figure 1 shows the distribution of the 211 field plots with respect to the TATR boundary and the six villages then located within the park. In order to depict the distribution of plots with respect to forest cover, the plots are overlaid on a map of the Normalized Difference Vegetation Index (NDVI) of the Landsat image. This index, whose values range from -1 to +1 , is derived from the red and infrared bands, and is an indicator of vegetation density (see [26]).

Nested circular plots were laid, with the outermost plot being 10 metres in radius. Within this, the species, diameter at breast height ( $\mathrm{dbh}$ ) and height were recorded for all trees (defined as individuals with dbh greater than $10 \mathrm{~cm}$ ). A nested sub-plot of 3 metres radius was used to record species, dbh and height for all shrubs and saplings (defined as individuals with dbh less than $10 \mathrm{~cm}$, but greater than one $\mathrm{cm}$ ). A further nested sub-plot of 1 meter radius was used to record the percentage of ground area covered by each herb-layer species. As the species found in the herb layer are highly seasondependent, and sampling was done during different seasons of the year, only tree and shrub/sapling data were used in this analysis for comparative purposes.

For each plot, we calculated the total species richness (based on tree and shrub/sapling nested plots), the tree species richness, the tree Shannon diversity and the number of trees [27]. Unlike temperate ecosystems where most remote sensing studies of vegetation diversity have taken place [11], and which tend to be dominated by a small number of species, this landscape is a subtropical ecosystem, and there are no species that are dominant in the tree canopy to the extent one can pick up from a satellite. The most common species in this region, Tectona grandis (teak), accounts for less than $18 \%$ of the total number of individuals in the tree canopy. Cassia fistula is the second most common species, and accounts for about $11 \%$ of all individuals. Further, these species are not dominant across all plots, but clustered and dominant in a few locations. Thus, using dominant tree species to derive correlations does not appear to be a suitable option for this ecosystem, unlike sometimes done for temperate ecosystems.

\subsubsection{Image data acquisition and pre-processing}

Landsat ETM+ (hereafter, Landsat) satellite imagery with a spatial resolution of $28.5 \mathrm{~m}$, acquired during 29th October 2001, was downloaded from the Global Land-cover Facility site hosted by the University of Maryland (glcfapp.umiacs.umd.edu). The Landsat image was georeferenced to five 1:50,000 scale Survey of India topographic maps covering our area of interest, using the nearest neighbor resampling algorithm [26]. Care was taken to ensure that the RMS error of image-to-image coregistration was less than half a Landsat pixel $(15 \mathrm{~m})$. Five IKONOS images were also acquired, covering the major portion of the park. The resolution of these images was $1 \mathrm{~m}$ (using PAN-sharpened multispectral data). As it was not possible to locate five images all taken on the same date, we purchased three adjacent images of a single satellite path taken on 5th December 2000, two images of an adjacent path taken on 27th December 2000, and finally, one adjacent image from a third satellite path taken on 22nd October 2000. All images are from the dry season, when it is easier to separate biomass in agriculture and grass fields from forests, and from dates that are closely spaced, thereby minimizing problems of image incompatibility due to seasonal and annual differences. 
Images taken from the same IKONOS satellite path on the same date did not require mosaicing. We atmospherically and radiometrically corrected images of adjacent paths, mosaiced them, and then registered the mosaiced images to the Survey of India 1:50,000 scale toposheets, with registration RMS errors maintained under $4 \mathrm{~m}$. This is essential to ensure that the association of the plot location with satellite data is not compounded by errors in co-registration. Finally, an overlay function and careful visual comparisons were used to verify that the Landsat and IKONOS images overlapped exactly, and misregistration was minimized [26]. All image processing was carried out using the ERDAS Imagine ${ }^{\mathrm{TM}}$ image processing software.

Each plot was located on the Landsat and IKONOS images using GPS coordinates taken at the plot center at the time of the field survey. The size of each plot (a $10 \mathrm{~m}$ radius circle) is less than the size of a Landsat ETM+ image pixel $(28.5 \mathrm{~m})$. Since this study was conducted within a forested landscape, however, GPS errors of up to $20 \mathrm{~m}$ were recorded in some instances, especially in those plots with a dense tree canopy. In order to account for these positional uncertainties, we instead associated each plot with a $3 \times 3$ window around the central pixel within which the plot was located, and calculated the average value for the 9 pixels located within this window (see also [28]). This was done for the $30 \mathrm{~m}$ bands 1-5 and 7, Tasseled Cap indices of Brightness, Greenness, and Wetness, and the NDVI, Infra Red Index (IRI), Middle Infra Red Index (MIRI), which are believed to relate to vegetation density and species diversity [11,26,29]. For the $15 \mathrm{~m}$ panchromatic band, we used a correspondingly larger $6 \times 6$ window. These image-derived values were related with the plant biodiversity values calculated for the associated plot. In addition, since species diversity can be influenced by landscape heterogeneity, we also computed the texture values for each index-here defined as the standard deviation of each index for a $3 \times 3$ window around the central pixel associated with each plot, or a $6 \times 6$ window for the $15 \mathrm{~m}$ panchromatic band - and related these values with plot biodiversity.

For the IKONOS data, the pixel resolution $(1 \mathrm{~m})$ is smaller than the plot size, which is a circle of $10 \mathrm{~m}$ radius. In order to account for GPS-related positional uncertainties of up to $20 \mathrm{~m}$, we used circles of $30 \mathrm{~m}$ radius ( $10 \mathrm{~m}$ plot radius plus $20 \mathrm{~m}$ maximum GPS uncertainties) which were drawn around the GPS coordinates of the plot center. For all pixels falling within the $30 \mathrm{~m}$ radius circle, the average and standard deviation of all pixels in bands 1-4, as the Tasseled Cap indices of Brightness, Greenness, and Wetness and the NDVI were computed and related with the relevant plot diversity and density/abundance data. Since IKONOS sensors do not record in the middle infra-red spectrum, we could not compute the IRI or MIRI for this dataset.

\subsection{Data Analysis: Relationship Between Spectral and Plant Biodiversity Data}

Pearson's correlation coefficients were used to evaluate the correlation between individual bands and indices derived from satellite image data, and field-derived species diversity information [30]. This provides us with an assessment of the potential of these single bands or indices and texture measures for alpha diversity assessment. We further applied a LOWESS (locally weighted scatterplot smoothing, also referred to as LOESS [31,32]) procedure to check for the trend of species diversity versus spectral information. LOWESS fits a polynomial function to a subset of the data, generally splitting the explanatory variable and giving a higher weight to points near the point where the response is being estimated. Notice that it is far beyond our aim to build a predictive model of species 
diversity based on only spectral variables (see the aim section). The intent of using LOWESS was to relax the application of "classical" least square based methods and to limit the use of fitting procedures to a graphical visualisation of the continuous variation of each species diversity variable with relation to spectral information [33]. All the analyses were performed by means of the R software ("cor" and "lowess" functions, package "stats", [34]).

\section{Results}

Tables 1 and 2 indicate the strength of correlation between each spectral and plant diversity variable, as assessed by the Pearson's correlation coefficient using Landsat ETM+ and Ikonos, respectively. A graphical comparison of each spectral variable versus total species richness, tree species richness, tree Shannon diversity and the number of trees is reported in Figures 2 to 5.

Table 1. Pearson correlation coefficients for species diversity vs. Landsat ETM+ spectral variables variables. Significance codes: $* * * p<0.001, * * \mathrm{p}<0.01, * \mathrm{p}<0.05$.

\begin{tabular}{|c|c|c|c|c|}
\hline Spectral variable & $\begin{array}{c}\text { Total species } \\
\text { richness }\end{array}$ & $\begin{array}{c}\text { Tree species } \\
\text { richness }\end{array}$ & $\begin{array}{c}\text { Tree Shannon } \\
\text { diversity }\end{array}$ & $\begin{array}{c}\text { Number of } \\
\text { trees }\end{array}$ \\
\hline Band 1 Mean & $-0.328 * * *$ & $-0.275 * * *$ & $-0.297 * * *$ & -0.066 \\
\hline Band 1 Standard Deviation & -0.118 & $-0.152 *$ & -0.125 & -0.112 \\
\hline Band 2 Mean & $-0.336 * * *$ & $-0.316^{* * *}$ & $-0.336 * * *$ & -0.105 \\
\hline Band 2 Standard Deviation & $-0.263 * * *$ & $-0.281 * * *$ & $-0.225 * * *$ & $-0.242 * * *$ \\
\hline Band 3 Mean & $-0.293 * * *$ & $-0.251 * * *$ & $-0.26 * * *$ & -0.091 \\
\hline Band 3 Standard Deviation & $-0.139 *$ & -0.131 & -0.129 & -0.045 \\
\hline Band 4 Mean & $-0.172 *$ & $-0.142 *$ & $-0.186^{* *}$ & 0.019 \\
\hline Band 4 Standard Deviation & $-0.146^{*}$ & -0.135 & $-0.167^{*}$ & -0.075 \\
\hline Band 5 Mean & $-0.246 * * *$ & $-0.255^{* * *}$ & $-0.268 * * *$ & -0.115 \\
\hline Band 5 Standard Deviation & $-0.137 *$ & $-0.142 *$ & $-0.151^{*}$ & -0.068 \\
\hline Band 7 Mean & $-0.254 * * *$ & $-0.25 * * *$ & $-0.26 * * *$ & -0.112 \\
\hline Band 7 Standard Deviation & -0.093 & -0.105 & -0.117 & -0.039 \\
\hline Panchromatic Band Mean & $-0.276 * * *$ & $-0.263 * * *$ & $-0.269 * * *$ & -0.133 \\
\hline Panchromatic Band Standard Deviation & -0.125 & -0.115 & $-0.138^{*}$ & -0.013 \\
\hline Brightness Mean & $-0.305 * * *$ & $-0.278 * * *$ & $-0.307 * * *$ & -0.077 \\
\hline Brightness Standard Deviation & $-0.181 * *$ & $-0.179 * *$ & $-0.179 * *$ & -0.108 \\
\hline Greenness Mean & $0.202 * *$ & $0.192 * *$ & $0.18 * *$ & 0.112 \\
\hline Greenness Standard Deviation & -0.101 & -0.107 & -0.123 & -0.007 \\
\hline Wetness Mean & $0.228 * * *$ & $0.241 * * *$ & $0.25 * * *$ & 0.118 \\
\hline Wetness Standard Deviation & -0.12 & -0.131 & $-0.142^{*}$ & -0.061 \\
\hline NDVI Mean & $0.166^{*}$ & $0.144 *$ & 0.125 & 0.087 \\
\hline NDVI Standard Deviation & -0.089 & -0.098 & -0.105 & -0.021 \\
\hline IRI Mean & 0.119 & $0.154 *$ & $0.138 *$ & 0.117 \\
\hline IRI Standard Deviation & -0.041 & -0.041 & -0.063 & 0.004 \\
\hline MIRI Mean & $0.22 * *$ & $0.193 * *$ & $0.193 * *$ & 0.092 \\
\hline MIRI Standard Deviation & 0.01 & -0.004 & -0.036 & 0.001 \\
\hline
\end{tabular}


Figure 2. Scatterplots of total species richness (SR) vs. Landsat ETM+ spectral variables. Fitted curves represent LOWESS based smoothing.
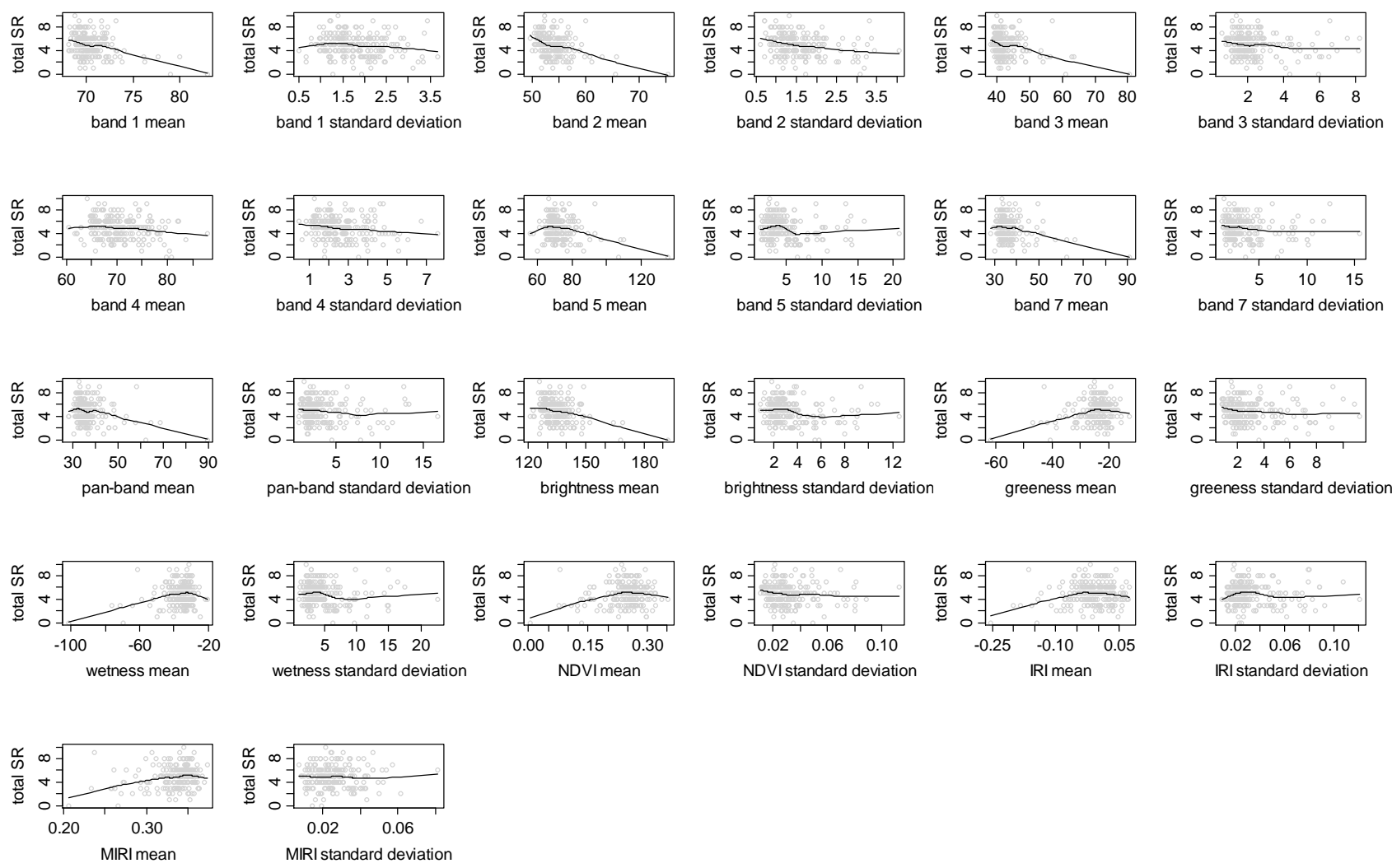

Figure 3. Scatterplots of tree species richness (SR) vs. Landsat ETM+ spectral variables. Fitted curves represent LOWESS based smoothing.
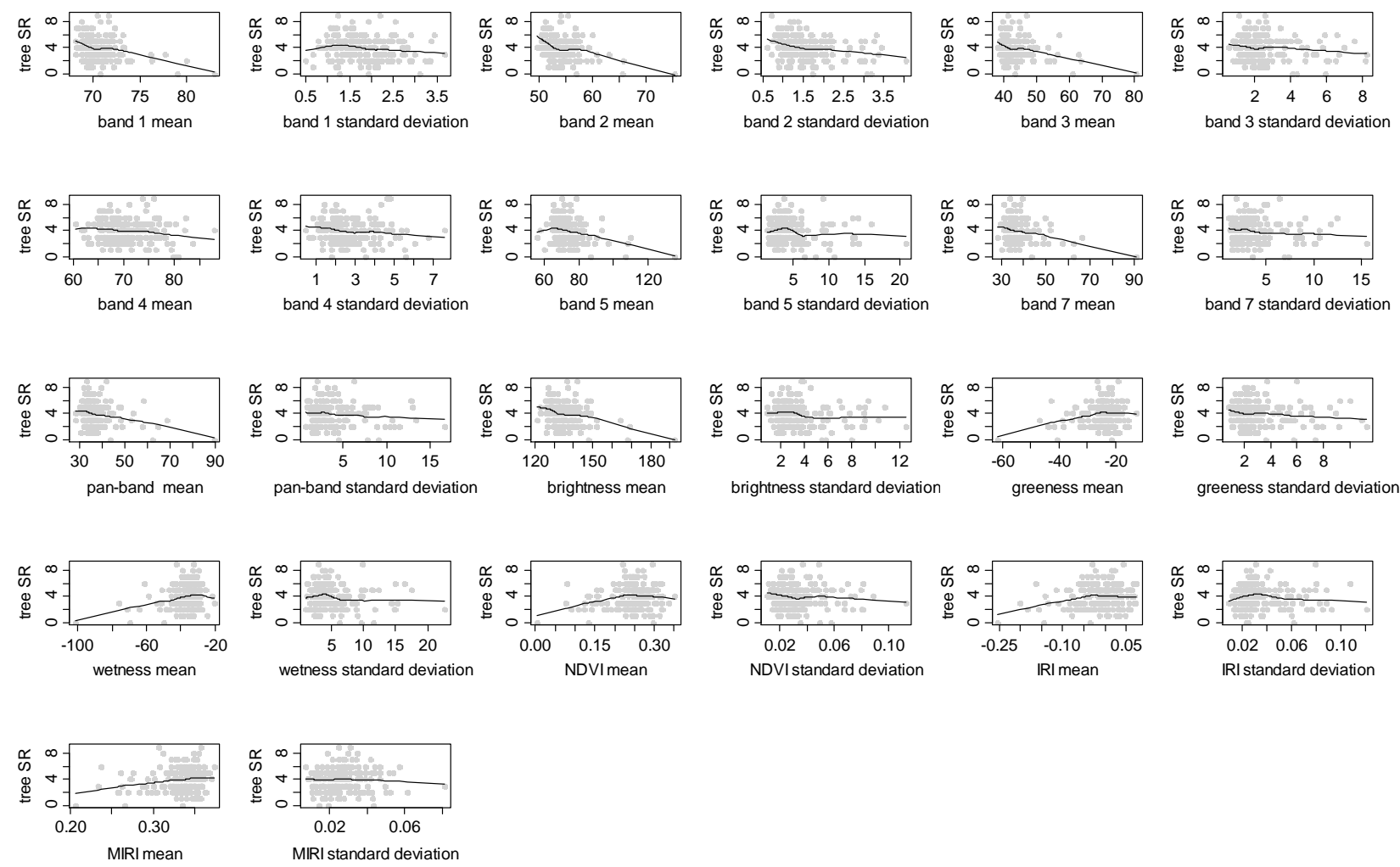
Figure 4. Scatterplots of tree Shannon diversity $v s$. Landsat ETM+ spectral variables Fitted curves represent LOWESS based smoothing.
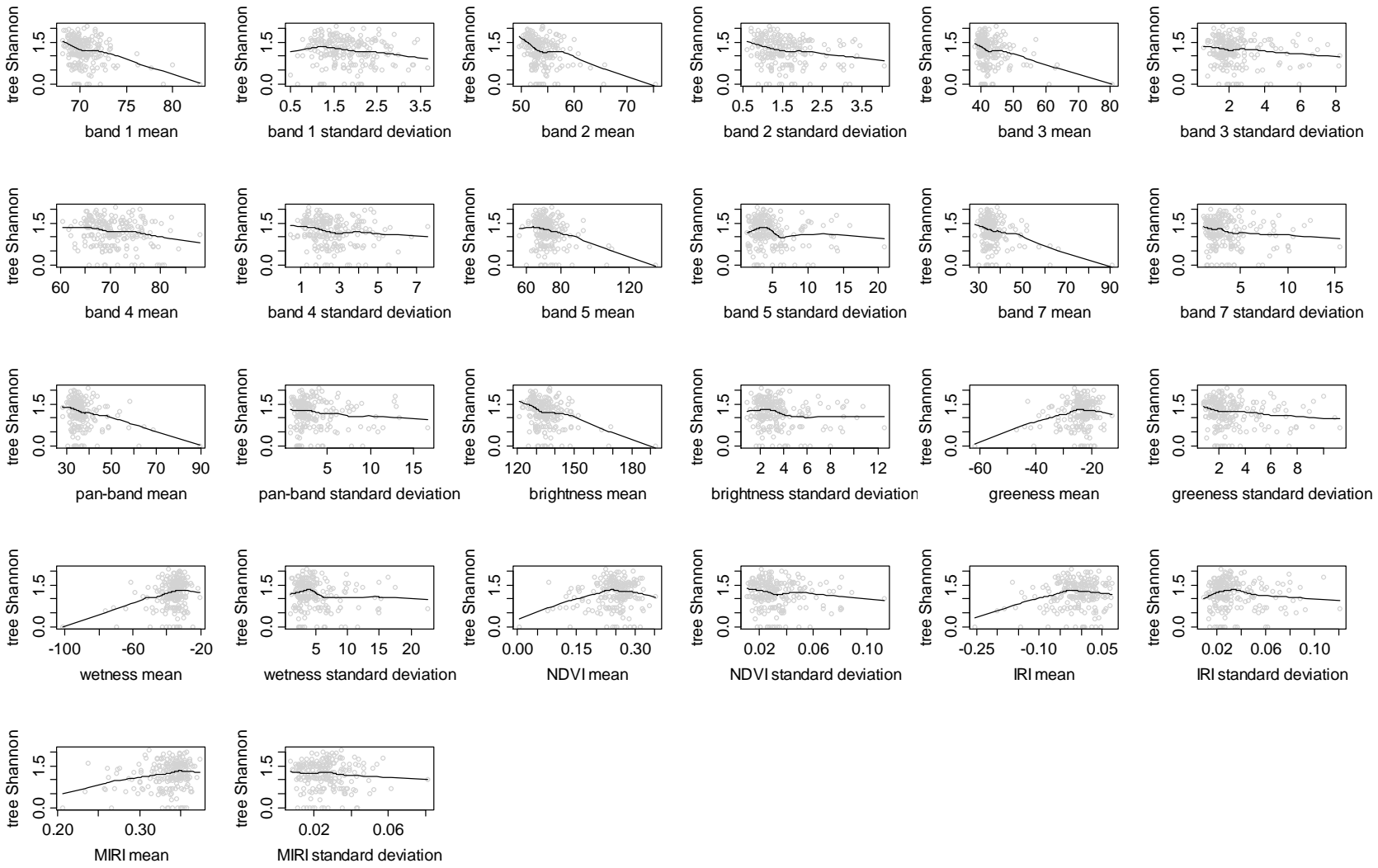

Figure 5. Scatterplots of the number of trees $v s$. Landsat ETM+ spectral variables Fitted curves represent LOWESS based smoothing.
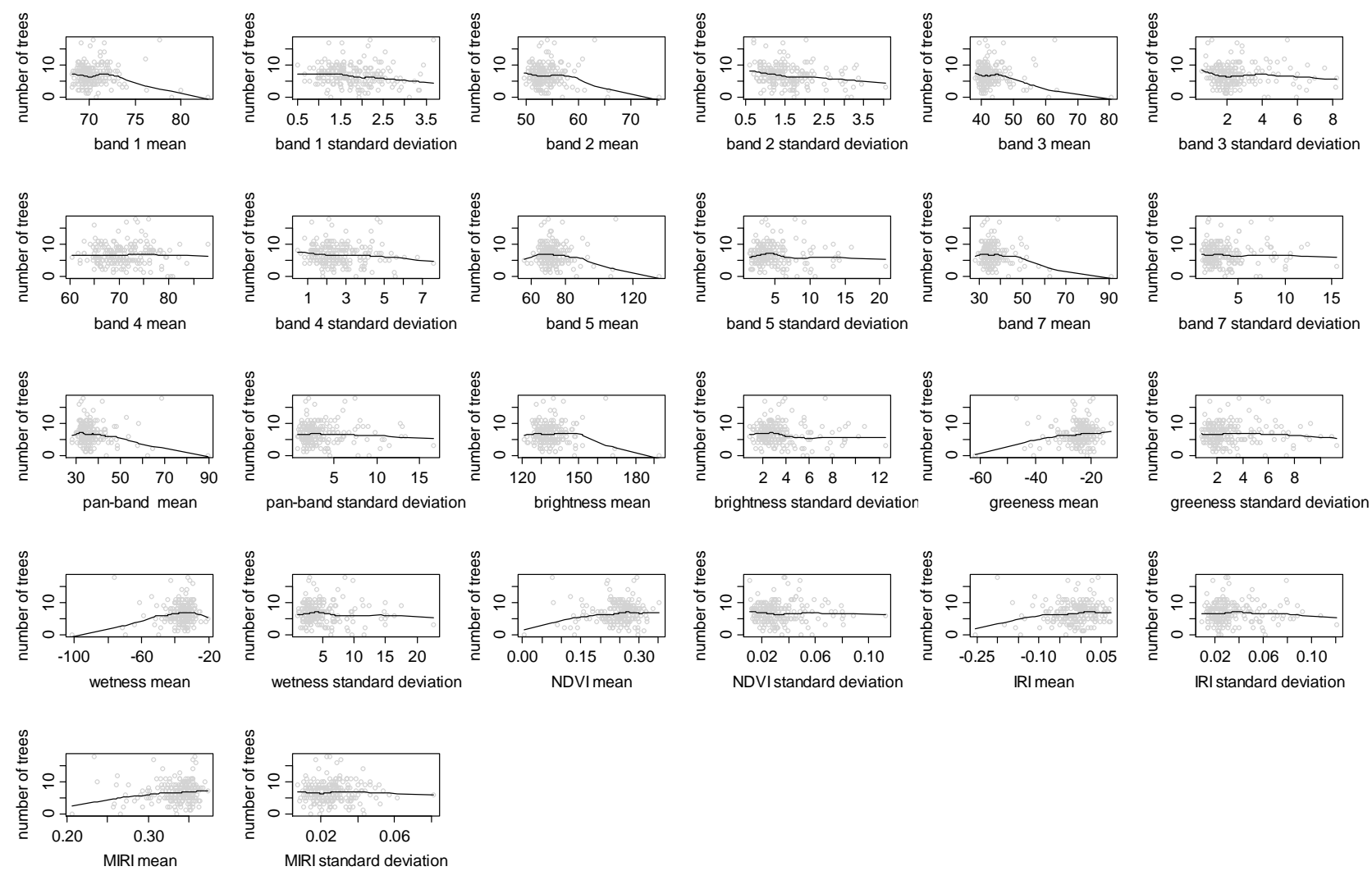
As Table 1 and Figure 5 indicate, Landsat ETM+ does not show high correlation with the number of trees in this dry subtropical ecosystem. Instead, the spectral information contained in the Landsat image appears most sensitive to total species richness (Figure 2, Table 1), followed by tree Shannon diversity (Figure 4, Table 1) and tree species richness (Figure 3, Table 1). Apart from band 4, all bands appear to pick up information on species richness and diversity, with bands 2 and 1 appearing to be the most sensitive (Table 1, Figures 2 to 5). Although the panchromatic band is significantly correlated to parameters of species richness and diversity, it is not any more sensitive than the $30 \mathrm{~m}$ bands, despite its greater spatial resolution of $15 \mathrm{~m}$ (Table 1, Figures 2-5, notice the flatness of the LOWESS curve of panchromatic variability versus all the considered species richness and diversity measures).

It is somewhat surprising to note the lower correlation coefficients and the lesser significance of the correlations between Landsat band 4 and plant diversity values (flat LOWESS curve of Figures 2 to 5 , see even Table 1), given the fact that this band is supposed to be receptive to the amount of vegetation in the upper canopy [26]. Possibly as a consequence of this, neither of the two most commonly used vegetation indices-NDVI nor IRI-appear very useful for plant diversity assessment in this ecosystem, while MIRI, which does not incorporate information from band 4, is more sensitive. Compared to all vegetation indices, however, Tasseled Cap indices, which incorporate information from all $30 \mathrm{~m}$ bands, appear to be more receptive to plant diversity. While all 3 tasseled cap indices appear to contain useful information, Brightness is the most sensitive to plant diversity levels, even more than Greenness. Greenness and Wetness means show significant positive relationships with most plant diversity indicators, signifying that — as can be expected — areas with greater vegetation biomass and more moisture tend to harbor increased levels of plant diversity in this dry tropical forest ecosystem. Brightness has a significant negative relationship with plant diversity and density, since open areas with greater levels of exposed soil and more brightness will naturally have decreased tree density and diversity.

Textural variables (as measured by standard deviation) do not appear to provide as much information on vegetation diversity. Texture provides absolutely no information significant for evaluating the number of trees. For most bands, texture provides either non-significant or weakly significant correlations to species richness and diversity, with the exceptions of the textural information contained in bands 2, 5 and Brightness. Textural variables are largely negatively correlated with plant diversity, indicating a decrease in plant diversity in heterogeneous areas that have been disturbed due to human action (largely biomass extraction, grazing and fire, in this ecosystem, see [25] for further details) in comparison to undisturbed and relatively homogeneous forest patches. In some landscapes, disturbance could lead to higher plant diversity due to pioneer species invading new niches created by fire or grazing, however this does not appear to be the case for this landscape. A similar pattern was found by Gillespie [39] in the same habitat (tropical dry forests) using NDVI. Quoting the author: "the higher the mean NDVI values and the lower the variation in NDVI values the higher the species richness". Two main causes appear to drive this process: (i) disturbance may result in significant structural damage to the tropical dry forest, and (ii) early successional forests generally contains lower species diversity while late-successional forests (with a higher biomass and a lower disturbance) generally contain a higher plant species diversity in terms of both richness and composition [39]. We even refer to Nagendra and Rocchini [7] for a complete dissertation on heterogeneity measurement in dry tropical forests. 
In contrast to Landsat, IKONOS imagery (Table 2 and Figures 6 to 9) appears less sensitive to plant diversity, with decreased strength of correlation as well as lowered statistical significance compared to the correlations observed with Landsat.

Table 2. Pearson correlation coefficients for species-diversity variables vs. IKONOS spectral variables. Significance codes: $* * * \mathrm{p}<0.001, * * \mathrm{p}<0.01, * \mathrm{p}<0.05$.

\begin{tabular}{lllll}
\hline Spectral variable & $\begin{array}{c}\text { Total species } \\
\text { richness }\end{array}$ & $\begin{array}{c}\text { Tree species } \\
\text { richness }\end{array}$ & $\begin{array}{c}\text { Tree Shannon } \\
\text { diversity }\end{array}$ & $\begin{array}{c}\text { Number of } \\
\text { trees }\end{array}$ \\
\hline Band 1 Mean & -0.018 & -0.041 & -0.012 & -0.131 \\
Band 1 Standard Deviation & -0.118 & -0.007 & -0.050 & $0.198^{* * *}$ \\
Band 2 Mean & -0.133 & $-0.148^{*}$ & -0.129 & $-0.159^{*}$ \\
Band 2 Standard Deviation & -0.127 & -0.021 & -0.066 & $0.183^{* * *}$ \\
Band 3 Mean & $-0.149^{*}$ & $-0.159^{*}$ & $-0.142^{*}$ & $-0.138^{*}$ \\
Band 3 Standard Deviation & $-0.140^{*}$ & -0.044 & -0.090 & $0.158^{*}$ \\
Band 4 Mean & $-0.235^{* * *}$ & $-0.149 *$ & $-0.189^{* *}$ & 0.016 \\
Band 4 Standard Deviation & -0.080 & 0.012 & -0.056 & $0.175^{*}$ \\
Brightness Mean & -0.080 & -0.008 & -0.050 & 0.131 \\
Brightness Standard Deviation & $-0.187^{* *}$ & -0.127 & $-0.175^{* *}$ & 0.051 \\
Greenness Mean & $-0.164 *$ & -0.069 & -0.110 & 0.086 \\
Greenness Standard Deviation & -0.068 & 0.026 & -0.049 & $0.166^{*}$ \\
Wetness Mean & $-0.169 *$ & $-0.192^{* *}$ & $-0.176^{* *}$ & -0.119 \\
Wetness Standard Deviation & -0.114 & -0.056 & -0.098 & 0.057 \\
NDVI Mean & -0.020 & 0.042 & 0.012 & 0.099 \\
NDVI Standard Deviation & -0.106 & 0.011 & -0.054 & $0.235^{* * *}$ \\
\hline
\end{tabular}

Figure 6. Scatterplots of total species richness (SR) vs. IKONOS spectral variables Fitted curves represent LOWESS based smoothing.
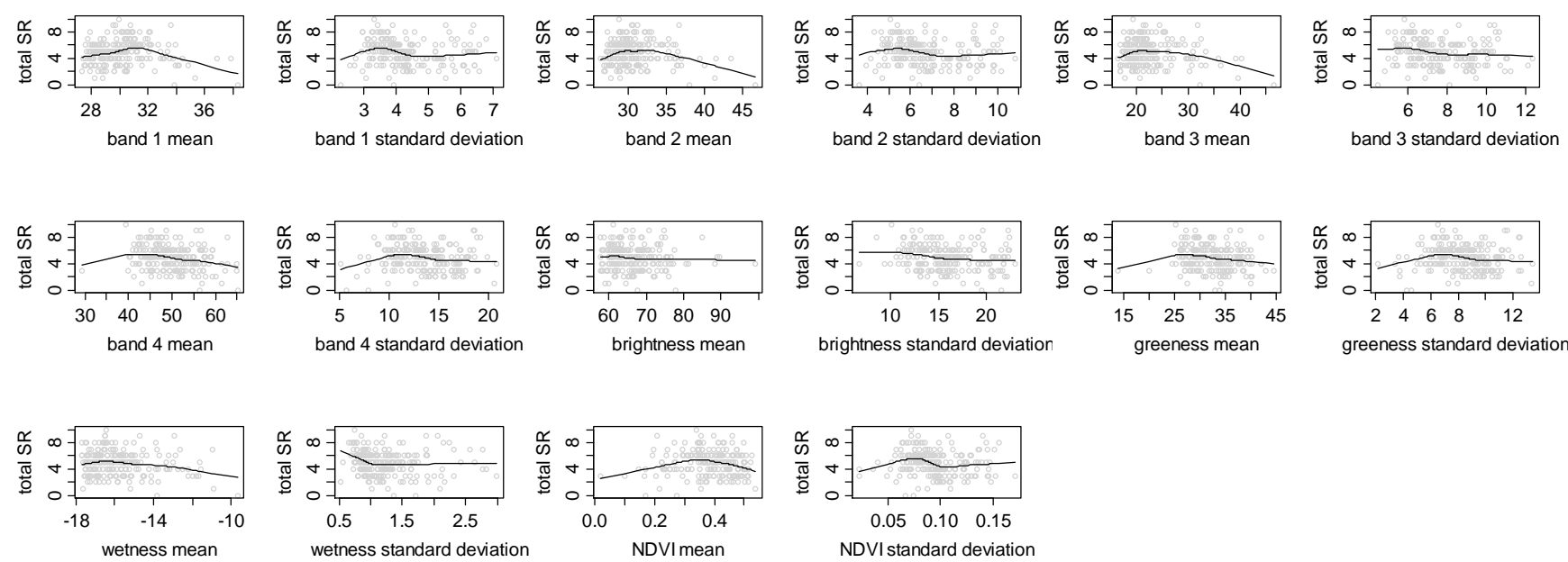
Figure 7. Scatterplots of tree species richness (SR) vs. IKONOS spectral variables. Fitted curves represent LOWESS based smoothing.
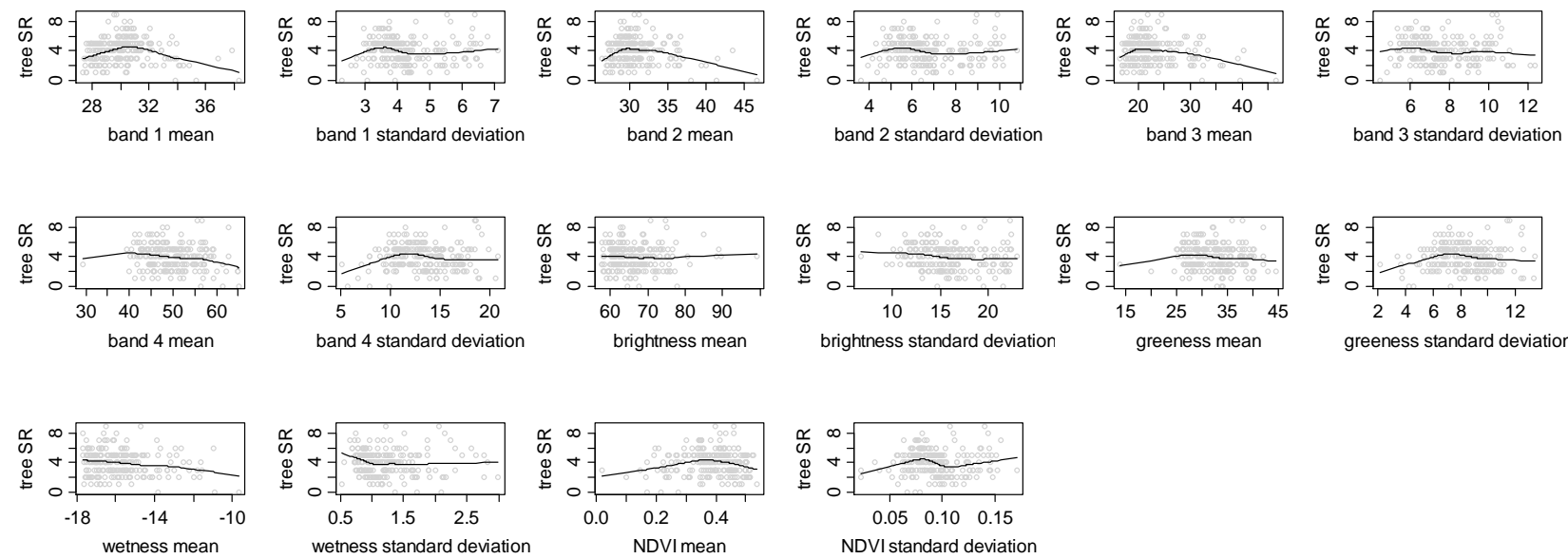

Figure 8. Scatterplots of tree Shannon diversity vs. IKONOS spectral variables. Fitted curves represent LOWESS based smoothing.
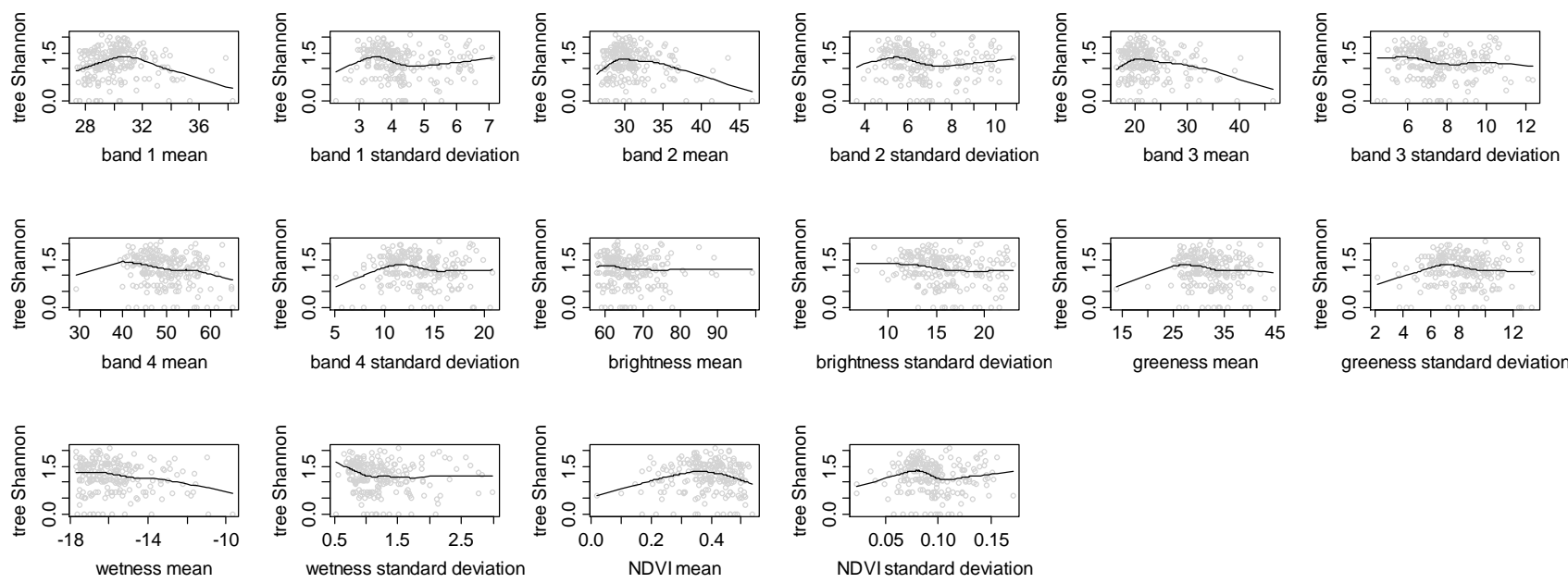

Figure 9. Scatterplots of the number of trees $v s$. IKONOS spectral variables. Fitted curves represent LOWESS based smoothing.
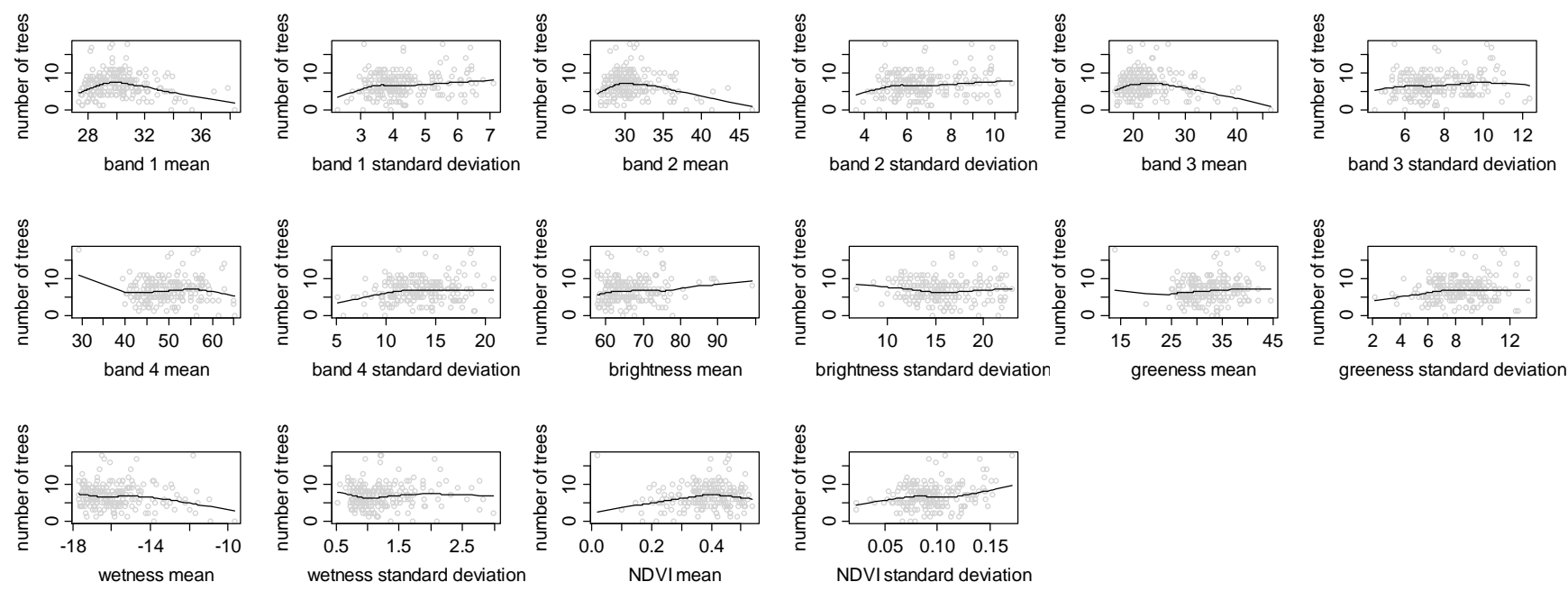
Interestingly, IKONOS is more sensitive to the number of trees than to plant diversity variables (Table 2, Figure 8). In this satellite, band 4 appears to be the best predictor of plant diversity, followed by the Tasseled Cap index of wetness, and band 3. Unlike with Landsat, mean values of Greenness are significantly negatively correlated with plant diversity. This may be a consequence of the fine scale variation in pixel values picked up by IKONOs, which encompasses shaded areas of tree cover and leads to an artefact of perceived reduction in greenness in vegetation-rich areas. Textural variables do not appear to be as sensitive to plant diversity information, apart from the brightness index, which as with Landsat, shows a negative correlation with plant diversity, possibly for similar reasons that heterogeneity indicates disturbance (e.g., fire or biomass uptake), which in this landscape appears to negatively impact vegetation diversity, since it structurally damages the tropical dry forest habitat.

\section{Discussion}

Through outlets such as Google Earth, high resolution satellite images have become increasingly popular, making detailed images of large parts of the Earth easily available to the larger public. Yet, the scientific applicability of these images remains limited due to technical issues ranging from calibration and geometric correction [35], to atmospheric correction [36], and spatial enhancement [37]. Due to these limitations, as well as the difficulty and expense related to acquiring these data, their use for ecological studies remains limited. This is particularly true in the tropics, where such data is not as easily available. Yet this study, one of the few field assessments of the utility of high resolution satellite data for vegetation diversity assessment in the tropics, clearly demonstrates that Landsat data, which are more readily available over all parts of the Earth, and which will soon be made available free to the global research community [7], appear to be more informative for purposes of plant diversity assessment.

The correlation coefficients observed between spectral data and field estimations of diversity at the plot level compare favourably overall with those noted in other studies [24,38,39]. It is somewhat puzzling to observe that, while Landsat derived vegetation indices of Greenness, NDVI and MIRI show a significant positive relationship with plant diversity (as expected and also as observed by other studies in similar landscapes, see [39]), the IKONOS derived Greenness index was significantly negatively correlated with plant diversity. We speculate that this may be an artifact of the fine resolution of IKONOS imagery, where a larger number of pixels in vegetation rich areas may be picking up numerous small patches of shade cast by vegetation canopies (see [7]), leading to lower perceived values of Greenness. Similar findings have been observed in a study conducted in a pine forest, where the IKONOS derived Enhanced Vegetation Index was found to have a negative relationship with the Leaf Area Index [40].

Although the maximum correlations achieved are less than 0.5 , the intent here was to compare different satellite platforms and not to use these imagery in themselves for absolute predictions. In fact it is unlikely that accurate predictions of vegetation species diversity can be completed using spectral variables alone, as even shown by the general flatness of LOWESS models. Instead, satellite-based variables may represent a set of good predictors within more complex models that include information on habitat types, soil, climate, and other variables such as autocorrelation $[11,41,42]$. 
Yet, overall trends clearly indicate that Landsat imagery appears to be better suited for assessing plant abundance and biodiversity compared to IKONOS data. This is largely due to the scale of data, which clearly makes a difference when deriving meaningful measures of landscape heterogeneity that relate to distributions of tree density and diversity. The scale of IKONOS data is too low for the purpose of plant diversity assessment in this landscape, with some of the $1 \mathrm{~m}$ pixels falling in tree shade, and others in sunlit areas. In contrast, Landsat imagery at the scale of $30 \mathrm{~m}$ appears more suited for the purpose of vegetation diversity assessment in this landscape.

As with other ecological data, the observation of plant biodiversity is scale dependent, and outcomes depend upon the spatial grain of study $[1,2,7,17]$. Ideally, the spatial resolution used should be such that information is obtained to an adequate degree of accuracy, using the least amount of data [11]. If the spatial resolution is too low, such that the size of a satellite image pixel is orders of magnitude less than the distribution of organisms (here, trees and higher plants), discrimination of organisms into different species or other categories becomes difficult. This is the aspect that has most often been emphasized in discussions of hyper spatial satellite imaging platforms, leading to the assumption that increasing image spatial resolution will always result in increased information on ecological distributions. For instance, Hernandez-Stefanoni and Dupuy [42] write that 'using a satellite image from a higher spatial resolution sensor like IKONOS could have yielded a more accurate estimation of species density, but would have been far more costly'.

Such implicit assumptions of the greater utility of high resolution satellite imagery are widespread, but do not always hold true. As an example, Rocchini [17] compared hyperspatial Quickbird (3 m pixel) against medium resolution Landsat (30 m pixel) imagery. He found that hyperspatial spectral data had similar correlations with species diversity compared to Landsat, which he attributed to the higher spectral resolution of Landsat data.

When spatial resolution is so high that pixels are at least an order of magnitude smaller than that of the object (here, organism) being categorized, then the variability of information provided by pixels covering a single object correspondingly increases, to the point that accurate identification can become very difficult. Data from platforms such as IKONOS, or aerial photography, have a spatial resolution high enough that pixels cover different parts of a tree, including shaded leaves, sunlit leaves, bark, and sometimes even understorey components and the forest floor [43-45]. The variation in spectral information across pixels covering different parts of a tree can be high enough to obscure the main pattern, thus making it difficult to assign pixels to species with any reasonable degree of accuracy. An ideal spatial resolution for biodiversity assessment would minimize the variance between spectral signatures of pixels covering a single organism or species, while maximizing the variation between organisms belonging to different species, as discussed further in Woodcock and Strahler [46] and Ricotta et al. [47].

Unlike many previous studies which have focused on the use of vegetation indices, we find that individual bands, particularly band 2 of Landsat and band 3 of IKONOS, provide critical additional information. Previous studies in evergreen landscapes $[29,48]$ indicate that Landsat derived indices of vegetation are highly sensitive to plant abundance, perhaps even more so than to species richness and Shannon diversity. Yet, we find in our study that vegetation indices have low, non significant relationships with stand density, while they demonstrate stronger relationships with species richness and diversity. This is in concordance with other reports that suggest that relationships between satellite 
derived vegetation indices and stand density vary from habitat to habitat. Two other studies in dry tropical forests have similar findings. Gillespie [39], studying tropical dry forests in southern Florida, found NDVI to be more strongly correlated with evergreen species density than deciduous, but more strongly correlated with deciduous species richness than evergreen. A study by Feeley et al. [24] in a dry tropical forest in Venezuela, also found vegetation indices (NDVI, IRI and MIRI) to be better correlated with species diversity indices than with stand density.

In congruence with studies in conifer forests in Scotland [49], and in tropical littoral forests in Madagascar [50], we find that textural measures derived from Landsat data-apart from band 2 texture - do not correlate with variations in stem density. Yet, several IKONOS derived measures of landscape texture - particularly band 1 texture, band 2 texture and NDVI texture - are significantly correlated with stem densities, indicating that the landscape microheterogeneity influential in driving stem density in this landscape appears to be at a scale closer to that of the $1 \mathrm{~m}$ IKONOS pixel than the $30 \mathrm{~m}$ Landsat pixel.

Landsat textural variables appear more useful in assessing vegetation diversity, however. Other research previously mentioned in dry tropical forests has indicated that Landsat derived NDVI textural measures help predict species diversity [39], as do findings from a tundra landscape in the Canadian arctic with substantial landscape heterogeneity [51]. In this landscape, where disturbance due to human impact by fire, grazing and biomass extraction frequently takes place [25], it is very likely that indices of texture are picking up areas of greater human disturbance, which leads to habitat microheterogeneity. While this may have led to increased plant diversity in other instances, here the impact appears to have been in the direction of reduced vegetation diversity. The scale of this heterogeneity appears to be important. Thus, we find several Landsat derived textural measures-particularly band 2 texture and Brightness texture-are negatively correlated with plant biodiversity variables. In contrast, few IKONOS derived textural variables appear to correlate with vegetation diversity. Thus, it appears that the finer spatial scales at which IKONOS derived measures of landscape heterogeneity are taken are better related to the scales at which stem density is distributed, while landscape heterogeneity at the Landsat pixel scales of $30 \mathrm{~m}$ appear more suited for the estimation of species richness and Shannon diversity distributions.

This study clearly demonstrates the utility of medium spatial resolution Landsat satellites for plant diversity assessment even in the species rich tropics. This is good news for ecologists and conservation biologists. Landsat data is available across the world, at regular intervals of time, and relatively low cost compared to high resolution images like IKONOS and Quickbird [7]. There are glaring gaps in the availability of hyperspatial data, especially prominent in tropical biodiversity hotspots, where the need for biodiversity assessment and monitoring is perhaps most critical [10,52,53].

Further, unlike medium resolution satellite platforms which routinely collect data across the globe at specified intervals, hyperspatial image collection is largely commissioned, making these data available less frequently, for fewer areas, and at irregular, often unpredictable intervals of time. Hyperspatial data is also very expensive. As previously stressed, Rocchini [17] has demonstrated the higher efficiency of Landsat imagery with respect to Quickbird, in terms of achieved results vs. costs. These costs put hyperspatial imagery out of the reach of many ecologists, especially those located in developing countries where the need is perhaps the greatest [7]. Finally, many countries also restrict the availability of high spatial resolution data, due to perceived issues of security [54]. Yet, 
unfortunately, while this study points to the greater utility of the long term Landsat platforms for biodiversity assessment, Landsat data of high quality is not available for most parts of the world after 2003, due to data striping issues with the Landsat sensor. Thus, at a time when it has just been announced that Landsat data will be made available free for all scientific use very soon, such research will only receive its final impetus when these data are available with the same frequency for recent time frames.

\section{Acknowledgements}

We thank the Maharashtra Forest Department for permissions to conduct field research, and the local communities living within and around Tadoba for their assistance. We also thank Gaurav Tyagi for assistance with data analysis. Financial support to HN from the Society in Science: Branco Weiss fellowship, ETH and the Ramanujan Fellowship from the Department of Science and Technology, Government of India, is gratefully acknowledged.

\section{References}

1. Kerr, J.T.; Ostrovsky, M. From space to species: ecological applications for remote sensing. Trends Ecol. Evol. 2003, 18, 299-305.

2. Carter, G.A.; Lucas, K.L.; Blossom, G.A.; Lassitter, C.L.; Holiday, D.M.; Mooneyhan, D.S.; Fastring, D.R.; Holcombe, T.R.; Griffith, J.A. Remote sensing and mapping of tamarisk along the Colorado river, USA: a comparative use of summer-acquired Hyperion, Thematic Mapper and QuickBird data. Remote Sens. 2009, 1, 318-329.

3. Prasad, P.R.C.; Nagabhatla, N.; Reddy, C.S.; Gupta, S.; Rajan, K.S.; Raza, S.H.; Dutt, C.B.S. Assessing forest canopy closure in a geospatial medium to address management concerns for tropical islands-Southeast Asia. Environ. Monit. Assess. 2009, doi: 10.1007/s10661-008-0717-4.

4. Wang, L.; Sousa, W.P.; Gong, P.; Biging, G. Comparison of IKONOS and QuickBird images for mapping mangrove species on the Caribbean coast of Panama. Remote Sens. Environ. 2004, 91, 432-440.

5. Everitt, J.J.; Fletcher, R.S.; Elder, H.S.; Yang, C. Mapping giant salvinia with satellite imagery and image analysis. Environ. Monit. Assess. 2008, 139, 35-40.

6. Duro, D.C.; Coops, N.C.; Wulder, M.A.; Han, T. Development of a large area biodiversity monitoring system driven by remote sensing. Prog. Phys. Geogr. 2007, 31, 253-260.

7. Nagendra, H.; Rocchini, D. Satellite imagery applied to biodiversity study in the tropics: the devil is in the detail. Biodiv. Conserv. 2008, 17, 3431-3442.

8. Nagendra, H.; Gadgil, M. Satellite imagery as a tool for monitoring species diversity: an assessment. J. Appl. Ecol. 1999, 36, 388-397.

9. Sanchez-Azofeifa, G.A.; Castro, K.L.; Rivard, B.; Kalascka, M.R.; Harriss, R.C. Remote sensing research priorities in tropical dry forest environments. Biotropica 2003, 35, 134-142.

10. Loarie, S.R.; Joppa, L.N.; Pimm, S.L. Satellites miss environmental priorities. Trends Ecol. Evol. 2007, 22, 630-632.

11. Nagendra, H. Using remote sensing to assess biodiversity. Int. J. Remote Sens. 2001, 22, 2377-2400. 
12. Turner, B.L., II; Lambin, E.F.; Reenberg, A. The emergence of land change science for global environmental change and sustainability. Proc. Natl. Acad. Sci. USA 2008, 104, 20666-20671.

13. Read, J.M.; Clark, D.B.; Venticinque, E.M.; Moreiras, M.P. Application of merged 1-m and 4-m resolution satellite data to research and management in tropical forests. J. Appl. Ecol. 2003, 40, 592-600.

14. Kovacs, J.M.; King, J.M.L.; de Santiago, F.F.; Flores-Verdugo, F. Evaluating the condition of a mangrove forest of the Mexican Pacific based on an estimated leaf area index mapping approach. Environ. Monit. Assess. 2008, doi: 10.1007/s10661-008-0523-z.

15. Everitt, J.J.; Yang. C.; Johnson, H.B. Canopy spectra and remote sensing of Ashe juniper and associated vegetation. Environ. Monit. Assess. 2007, 130, 403-413.

16. Levin, N.; Shmida, A.; Levanoni, O.; Tamari, H.; Kark, S. Predicting mountain plant richness and rarity from space using satellite-derived vegetation indices. Divers. Distrib. 2007, 13, 692-703.

17. Rocchini, D. Effects of spatial and spectral resolution in estimating ecosystem $\alpha$-diversity by satellite imagery. Remote Sens. Environ. 2007, 111, 423-434.

18. Warren, A.J.; Collins, M.J. A pixel-based semiempirical system for predicting vegetation diversity in boreal forest. Int. J. Remote Sens. 2007, 28, 83-105.

19. Curran, P.J. Remote sensing of foliar chemistry. Remote Sens. Environ. 1989, 30, 271-278.

20. Martin, M.E.; Aber, J.D. High spectral resolution remote sensing of forest canopy lignin, nitrogen, and ecosystem processes. Ecol. Appl. 1997, 7, 431-443.

21. Cochrane, M.A. Using vegetation reflectance variability for species level classification of hyperspectral data. Int. J. Remote Sens. 2000, 21, 2075-2087.

22. Clark, M.; Roberts, D.A.; Clark, D.B. Hyperspectral discrimination of tropical rain forest tree species at leaf to crown scales. Remote Sens. Environ. 2005, 96, 375-398.

23. Leimgruber, P.; Christen, C.A.; Laborderie, A. The impact of Landsat satellite monitoring on conservation biology. Environ. Monit. Assess. 2005, 106, 81-101.

24. Feeley, K.J.; Gillespie, T.W.; Terborgh, J.W. The utility of spectral indices from Landsat ETM+ for measuring the structure and composition of tropical dry forests. Biotropica 2005, 37, 508-519.

25. Nagendra, H.; Pareeth, S.; Ghate, R. People within parks: Forest villages and fragmentation in the Tadoba-Andhari Tiger Reserve, India. Appl. Geogr. 2006, 26, 96-112.

26. Jensen, J.R. Remote Sensing of the Environment: An Earth Resource Perspective; Prentice Hall: New Jersey, NJ, USA, 2000.

27. Legendre, P.; Legendre, L. Numerical Ecology, Second English Edition; Elsevier Science BV: Amsterdam, The Netherlands, 1998.

28. Oza, M.P.; Srivastava, V.K.; Devaiah, P.K. Estimating tree volume in tropical dry deciduous forest from Landsat TM data. Geocarto Int. 1996, 1, 33-39.

29. Bawa, K.; Rose, J.; Ganeshaiah, K.N.; Barve, N.; Kiran, M.C.; Umashaankar, R. Assessing biodiversity from space: an example from the Western Ghats, India. Conserv. Ecol. 2002, 6, 7. Available online at: http://wwwconsecolorg/vol6/iss2/art7 (accessed on 9 May 2008).

30. Sokal, R.R.; Rohlf, F.J. Biometry, 3rd ed.; Freeman: New York, NY, USA, 1995.

31. Cleveland, W.S. Robust locally weighted regression and smoothing scatterplots. J. Am. Stat. Assoc. 1979, 74, 829-836. 
32. Cleveland, W.S.; Devlin, S.J. Locally weighted regression: an approach to regression analysis by local fitting. J. Am. Stat. Assoc. 1988, 83, 596-610.

33. Crawley, M.J. Statistics: An Introduction Using R; Wiley \& Sons: Chichester, UK, 2005.

34. $R$ Development Core Team. R: A Language and Environment for Statistical Computing; $\mathrm{R}$ Foundation for Statistical Computing: Vienna, Austria, 2008.

35. Fraser, C.S.; Dial, G.; Grodecki, J. Sensor orientation via RPCs. ISPRS J. Photogramm. Remote Sens. 2006, 60, 182-194.

36. Wu, J.; Wang. D.; Bauer, M.E. Image-based atmospheric correction of QuickBird imagery of Minnesota cropland. Remote Sens. Environ. 2005, 99, 315-325.

37. Sohn, G.; Dowman, I. Data fusion of high-resolution satellite imagery and LiDAR data for automatic building extraction. ISPRS J. Photogramm. Remote Sens. 2007, 62, 43-63.

38. Jakubauskas, M.E.; Price, K.P. Empirical relationships between structural and spectral factors of Yellowstone Lodgepole Pine forests. Photogramm. Eng. Remote Sens. 1997, 63, 1375-1381.

39. Gillespie, T.W. Predicting woody-plant species richness in tropical dry forests: a case study from South Florida, USA. Ecol. Appl. 2005, 15, 27-37.

40. Che, X.; Vierling, L.; Rowell, E.; DeFelice, T. Using lidar and effective LAI data to evaluate IKONOS and Landsat $7 \mathrm{ETM}+$ vegetation cover estimates in a ponderosa pine forest. Remote Sens. Environ. 2004, 91, 14-26.

41. Gillespie, T.W.; Foody, G.M.; Rocchini, D.; Giorgi, A.P.; Saatchi, S. Measuring and modeling biodiversity from space. Prog. Phys. Geogr. 2008, 32, 203-221.

42. Hernandez-Stefanoni, J.L.; Dupuy, J. Mapping species density of trees, shrubs and vines in a tropical forest, using field measurements, satellite multispectral imagery and spatial interpolation. Biodiv. Conserv. 2007, 16, 3817-3833.

43. Gougeon, F.A. Comparison of possible multispectral classification schemes for tree crowns individually delineated on high spatial resolution MEIS images. Can. J. Remote Sens. 1995, 21, 1-9.

44. Biging, G.S.; Dobbertin, M.; Murphy, E.C. A test of airborne multispectral videography for assessing the accuracy of wildlife habitat maps. Can. J. Remote Sens. 1995, 21, 357-366

45. Meyer, P.; Staenz, K.; Itten, K.I. Semi-automated procedures for tree species identification in high spatial resolution data from digitised colour infrared-aerial photography. ISPRS J. Photogramm. Remote Sens. 1996, 51, 5-16.

46. Woodcock, C.E.; Strahler, A.H. The factor of scale in remote sensing. Remote Sens. Environ. 1987, 21, 311-332.

47. Ricotta, C.; Avena, G.C.; Volpe, F. The influence of principal component analysis on the spatial structure of a multispectral dataset. Int. J. Remote Sens. 1999, 20, 3367-3376.

48. Freitas, S.R.; Mello, M.C.S.; Cruz, C.B.M. Relationships between forest structure and vegetation indices in Atlantic Rainforest. Forest Ecol. Manage. 2005, 218, 353-362.

49. Puhr, C.B.; Donoghue, D.N.M. Remote sensing of upland conifer plantations using Landsat TM data: A case study from Galloway, south-west Scotland. Int. J. Remote Sens. 2000, 21, 633-646.

50. Ingram, J.C.; Dawson, T.P.; Whittaker, R.J. Mapping tropical forest structure in southeastern Madagascar using remote sensing and artificial neural networks. Remote Sens. Environ. 2005, 94, 491-507. 
51. Gould, W. Remote sensing of vegetation, plant species richness, and regional biodiversity hotspots. Ecol. Appl. 2000, 10, 1861-1870.

52. Goetz, S. Crisis in Earth observation. Science 2007, 315, 1767.

53. Kark, S.; Levin, N.; Phinn, S. Global environmental priorities: making sense of remote sensing: reply to TREE Letter: satellites miss environmental priorities by Loarie et al. (2007). Trends Ecol. Evol. 2008, 23, 181-182.

54. Srikantia, S.V. Restriction on maps: a denial of valid geographic information. Curr. Sci. 2000, 79, 484-488.

(C) 2010 by the authors; licensee Molecular Diversity Preservation International, Basel, Switzerland. This article is an open-access article distributed under the terms and conditions of the Creative Commons Attribution license (http://creativecommons.org/licenses/by/3.0/). 